\title{
A DESCRIPTIVE THEORY OF THE PHOTO DE-ACTIVATION OF SILVER IODIDE AS AN ICE-CRYSTAL NUCLEUS
}

\author{
By N. H. Fletcher \\ C.S.I.R.O. Radiophysics Laboratory, Sydney, Australia \\ (Original manuscript received 30 September 1958; revised manuscript received 16 January 1959)
}

\section{ABSTRACT}

The photo de-activation of silver iodide as an ice-crystal nucleus is ascribed to the production of photolytic silver at trapping centers in the volume and on the surface of the nucleating particle. Silver produced at the surface raises the free energy of the interface between the nucleus and ice and so reduces the activity of the particle. The rate of de-activation is found to depend upon the size of the particle, and thus the size distribution of particles in a smoke has a strong influence upon its decay behavior. Calculations on the basis of this theory explain the wide variations in the decay rate reported in different experiments.

\section{Introduction}

Experiments $[1 ; 2 ; 3]$ have shown that the effectiveness of silver iodide as an ice-crystal nucleus decreases with time in the free atmosphere, and this decay has been traced [2 through 8$]$ to the influence of the ultraviolet component of sunlight which is strongly absorbed by the silver-iodide particles. There is, however, little agreement as to the magnitude of this effect, estimates ranging from a decrease in the number of active particles by less than a factor of 2 per hour [6], to a decrease of as much as a factor $10^{9}$ per hour [5] under comparable conditions of illumination. These disagreements are in most cases well outside the limits of experimental uncertainty and appear to be related in some way to the methods used for the preparation of smoke samples.

Whilst it can be seen in a general way that ultraviolet light reduces $\mathrm{AgI}$ to metallic silver, and in doing so must adversely affect its nucleating properties, no detailed theory of this process has hitherto been proposed. The present paper aims to describe this deactivation process in some detail so that the relevant physical parameters become apparent. This is treated in a quite general way, so that the results hold for any nucleating substance which is decomposed by radiation. After deriving this general result we shall apply the theory explicitly to the case of silver iodide.

\section{The mechanism of de-activation}

Silver iodide is a good ice-crystal nucleus for three reasons. Firstly, its crystal surface potential has approximately the same symmetry and periodicity as the ice lattice; secondly, this surface potential is such that the binding energy of $\mathrm{H}_{2} \mathrm{O}$ molecules on the surface reduces the interfacial free energy to a very low value. The third reason; which we hope to discuss in a forthcoming publication, relates to the relative positions of positive and negative ions in the lattice, and will not concern us here.

Details of the behavior of AgI particles as icecrystal nuclei have been given in another publication [9], where it is shown that AgI acts as a sublimation rather than as a freezing nucleus under ordinary circumstances. It has also been possible to find the relation between the radius of an AgI particle and the temperature at which it becomes active as a sublimation nucleus $[9 ; 10]$. This relation is shown graphically in fig. 1 and will form the basis of much of what follows. Similar curves can, in principle, be determined for other nucleating agents, whether they act as sublimation or as freezing nuclei.

When an $\mathrm{AgI}$ crystal is irradiated with light having a wavelength within its fundamental absorption band, photo electrons are produced. A certain number of these electrons may recombine directly with holes, but many move freely through the crystal until they become

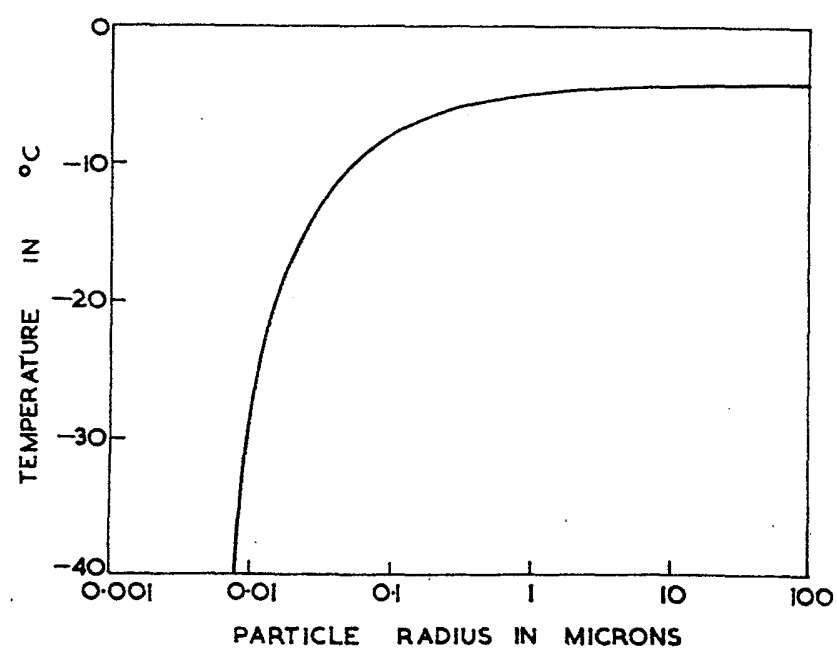

FIG. 1. Temperature at which a silver-iodide particle of given radius will nucleate an ice crystal in one second from an environment at water saturation. 
trapped at some sort of crystal imperfection. The silver ions in the AgI lattice have a very high mobility and are attracted by the field of the trapped electron. One of these ions ultimately combines with the trapped electron to produce a silver atom in the vicinity of the trap. A review of the mechanism of the photolytic process which we have outlined here may be found in one of the several such articles by Mitchell [11].

The photolytic silver produced at trapping centers within the bulk of the crystal has no effect upon its nucleating properties, but that produced at the surface must certainly influence the activity. Mitchell [11] has shown that surface silver builds up in impure crystals by the production of very many small deposits of two or three silver atoms rather than by the growth of just a few very large clusters. The production of surface silver can thus be regarded as causing a continuous change in surface properties and a corresponding continuous change in the interfacial free energy. Ultimately, of course, the surface will collapse to the silver lattice with a corresponding abrupt change in properties, but well before this the nucleating efficiency will have fallen to a very low value.

We shall now treat the various stages of this process in as nearly a quantitative manner as possible so that the magnitude of the effect and the relevant physical parameters become apparent.

Consider a spherical particle of radius $R$ upon which is incident radiation of density $I$ quanta $\mathrm{cm}^{-2} \mathrm{sec}^{-1}$ in its fundamental absorption band. The number of quanta absorbed per second can be written $I F(k, R)$ where $k$ is the absorption coefficient in the band and $F(k, R)$ represents the absorption cross section. The form of this function will be discussed later. If the quantum efficiency for production of photo electrons which later give rise to silver atoms ${ }^{1}$ is $\alpha$, then the rate of production of such electrons is

$$
(d E / d t)_{I}=\alpha F(k, R) I .
$$

These electrons become caught in traps and produce silver atoms. If the density of traps is $\beta \mathrm{cm}^{-3}$, then the rate of removal of electrons in the volume is given by

$$
(d E / d t)_{V}=-\beta E X,
$$

where $X$ is a constant involving the cross section of a trap and the thermal velocity of the electrons.

Some electrons, however, are caught at surface traps whose density is $\gamma \mathrm{cm}^{-2}$, giving

$$
(d E / d t)_{S}=-\gamma S(E / V) X
$$

where $S$ is the surface area and $V$ is the volume of the particle. For a sphere, this gives

\footnotetext{
I This quantum efficiency is a composite concept which involves the cross section and thermal stability of the electron traps and the rate at which silyer ions move through the lattice to combine with these electrons. It is, however, a readily measurable physical quantity.
}

$$
(d E / d t)_{S}=-3 \gamma E X / R .
$$

In the steady state, of course,

$$
d E / d t=0
$$

so that combining equations (1), (2), (4), and (5),

$$
\alpha F(k, R) I=\beta E X+3 \gamma E X / R
$$

or

$$
E=\frac{\alpha F(k, R) I}{(\beta+3 \gamma / R) X}
$$

Thus, from (4),

$$
\left(\frac{d E}{d t}\right)_{S}=-\frac{3 \alpha \gamma F(k, R) I}{\beta R+3 \gamma} .
$$

Now the rate of increase of density of surface silver atoms is just

$$
\frac{d n}{d t}=-\frac{1}{4 \pi R^{2}}\left(\frac{d E}{d t}\right)_{S}
$$

whence

$$
\frac{d n}{d t}=\frac{3 \alpha \gamma F(k, R) I}{4 \pi R^{2}(\beta R+3 \gamma)} .
$$

Returning now to the nucleation problem, the behavior of a surface depends $[10]$ on the parameter

$$
m=\left(\sigma_{13}-\sigma_{23}\right) / \sigma_{12},
$$

where $\sigma$ denotes surface free energy and subscript 1 refers to air saturated with vapor, 2 to ice, and 3 to the nucleating particle. If the geometry of the surface remains unchanged except that isolated silver atoms are added to it, then $\sigma_{23}$ and $\sigma_{13}$ will increase linearly with the density $n$ of these added atoms. Thus the parameter $m$ varies linearly with $n$.

To relate changes in $m$ to changes in the supercooling $\Delta T$ required to nucleate ice crystals from an environment at water saturation, we examine the calculated curves for various values of $m$ given in reference $[10]$. From these, we see that, at any rate in the temperature range 0 to $-40 \mathrm{C}, \Delta T$ varies approximately linearly with $(1-m)$.

Combining these two steps then, we have

$$
\dot{d} \Delta T / d t=A d n / d t,
$$

where $A$ is a constant which must be evaluated for specific cases. We shall see later how this can be done.

Now, combining equations (10) and (12), we have

$$
\frac{d \Delta T}{d t}=\frac{3 A \alpha \gamma F(k, R) I}{4 \pi R^{2}(\beta R+3 \gamma)} .
$$

This equation describes the decline in activity of a given particle. The rate depends upon the material of the particle through the parameters $A, \alpha$, and $k$ and upon its purity and crystal perfection through parame- 
ters $\alpha, \beta$, and $\gamma$. The rate of decrease of activity depends also upon the particle radius $R$ and upon the incident radiation intensity, $I$.

Smokes generally contain a large range of particle sizes so that their behavior is more complex than that of a single particle. They can, however, be treated by breaking them into small size ranges which can then be treated as units. In a later section we shall perform this operation for some typical smokes.

Before proceeding with this discussion, we must evaluate more explicitly some of the factors appearing in our general expression (13). We now restrict ourselves to the case of silver iodide and shall confine our attention to this particular case for the remainder of the paper.

\section{Explicit parameters for AgI}

Absorption cross section. - The evaluation of absorption cross sections for particles comparable in size with the wavelength of light is a very complicated procedure. However, an example worked out by van de Hulst [12] shows that, for substances like $\mathrm{AgI}$ in its fundamental absorption band, a treatment on strictly geometric lines is correct within a factor 2. We shall therefore use this approach.

From data given in the International Critical Tables, the absorption coefficient, $k$, of silver iodide is approximately $1 \times 10^{5} \mathrm{~cm}^{-1}$ for wavelengths less than about $4100 \AA$. A simple geometric treatment of absorption, neglecting reflection and scattering, gives

$$
F(k, R)=\pi R^{2}-\frac{\pi}{2 k^{2}}\left[1-(2 k R+1) e^{-2 k R}\right] .
$$

If $k R \gg 1$, then $F(k, R)=\pi R^{2}$ and absorption is confined to layers near the surface. If $k R \ll 1$, then $F(k, R)=4 \pi R^{3} k / 3$, as is readily shown by expanding the exponential, and absorption is throughout the volume of the particle. The transition between these two forms is near $R=10^{-5} \mathrm{~cm}$ which is well within our range of interest.

Quantum effciency.-Seitz [13] quotes values for the quantum efficiency for production of photolytic silver in $\mathrm{AgCl}$ and $\mathrm{AgBr}$ as 0.4 and 0.33 , respectively. In the absence of other information, we may therefore assume a quantum efficiency of $\alpha=0.3$ for $\mathrm{AgI}$. It is to be expected that this value decreases in the later stages of photolysis when the silver concentration becomes appreciable, but we shall neglect this effect here.

The value of $\alpha$ may also be decreased through the presence of traps which act as recombination centers for electrons and holes, without the production of photolytic silver. In the absence of more detailed knowledge of the trapping mechanism in AgI, we cannot say what these traps may be, but it should be recognized that $\alpha$ will be, to some extent, a structuresensitive property.

Radiation intensity.-Since most practical interest centers upon the decay of silver-iodide smoke in natural illumination, we require a typical value for the active component of daylight. Tables given by Koller [14] show that the maximum intensity of sunlight near the ground amounts to between $10^{15}$ and $10^{16}$ quanta $\mathrm{cm}^{-2} \mathrm{sec}^{-1}$ in the fundamental absorption band of AgI. Allowing for deviations of a few hours from noon (about a factor 2) and for changes of season (as much as a factor 6), typical values for clear days probably lie between $10^{14}$ and $10^{15}$ quanta $\mathrm{cm}^{-2} \mathrm{sec}^{-1}$. Any cloud will, of course, lower this value quite considerably, and since many experiments (for example, [2]) were made with appreciable cloud cover, we shall adopt, as a typical value for I, $10^{14}$ quanta $\mathrm{cm}^{-2} \mathrm{sec}^{-1}$. This is also of the same order of magnitude as the intensity produced by the ultraviolet lamps used in some of the laboratory experiments referred to.

It must, of course, be recognized that the decay rate depends critically upon the radiation intensity, in that the integrated exposure $I t$ is the real quantity of importance. Results may thus vary widely on different occasions.

Trap densities.-As we remarked before, traps are associated with foreign atoms and with crystal imperfections. We have denoted volume trap density by $\beta$ and surface trap density by $\gamma$. We shall now find a relation between these two quantities and estimate their magnitudes.

We can write

$$
\gamma=\gamma_{0}+\epsilon \beta
$$

where $\gamma_{0}$ represents traps of an essentially surface nature, and $\epsilon \beta$ represents volume traps which happen to lie in the surface. $\epsilon$ is clearly just the ratio of the number of molecules per unit of surface area to the number of molecules per unit volume. For AgI, there are $1.4 \times 10^{22}$ molecules $\mathrm{cm}^{-3}$ so that $\epsilon$ has the value $\left(1.4 \times 10^{22}\right)^{-\frac{1}{3}}=4 \times 10^{-8} \mathrm{~cm}$.

There is evidence $[11 ; 13]$ that a perfect surface does not contribute any traps, so that in the absence of any adsorbed impurities $\gamma_{0} \approx 0$ and

$$
\gamma \approx \epsilon \beta=4 \times 10^{-8} \beta
$$

We observe that in this case the absolute magnitude of $\beta$ does not appear in the final result, since only the ratio $\gamma / \beta$ is involved in (13).

For our assumptions to be valid, we require a reasonable density of traps on even the smallest crystal considered. This implies at least one trap to the area covered by a critical embryo. Since a critical embryo is about $100 \AA$ in diam in all the cases we consider, this implies a $\beta$ value of about $2 \times 10^{19} \mathrm{~cm}^{-3}$. This corresponds to a trap density of about 0.1 per 
cent. This amount of impurity is almost certain to be present in the particles we are considering, especially since most of them are produced by some sort of heating or burning. It is also likely that non-stoichiometry produces trapping centers, so it is probably not essential to have as high a percentage as this of foreign atoms.

Seitz [13] estimates the density of traps associated with lattice defects in pure, well annealed crystals of $\mathrm{AgCl}$ or $\mathrm{AgBr}$ to be about $10^{14} \mathrm{~cm}^{-3}$. The density of such traps is likely to be several orders of magnitude greater than this, however, in the case of $\mathrm{AgI}$, because of the phase change which occurs near $146 \mathrm{C}$ as the crystals are cooled. Traps generated in this way may thus make a significant contribution to $\beta$.

We shall initially make the assumption, discussed above, that $\gamma_{0}=0$, and use equation (16) in our calculations. This implies that there has been no adsorption and no surface chemical attack during formation of the AgI particles.

Solarization.-Mention should be made of one further effect which we shall have to ignore in the present calculation. When metallic silver is formed within a crystal, iodine is liberated and migrates to the crystal surface. In doing so, it may encounter clumps of silver atoms with which it can recombine, destroying the photolytic precipitate. Because of the smaller free energy of formation of AgI, this is less likely to happen with this salt than with the bromide or chloride. It is, however, a possible reaction.

Its effect would be to decrease the rate of silver production, especially at the crystal surface, and hence to slow the deactivation process. Because of the surface to volume ratio, the effect would be greater for large crystals than for small ones.

Unfortunately we have, as yet, too little detailed nformation to treat this process quantitatively, and must agree to ignore it in the present discussion.

De-activation constant. - We must now attempt to evaluate the constant $A$, appearing in equation (12), which relates the appearance of photolytic silver to the increase in critical supercooling. There are, in principle, two ways in which this can be done.

It is possible, in principle, to calculate the increase in interfacial free energy caused by replacing one surface ion by a silver atom. This involves a fairly precise knowledge of the molecular constants involved. Equa-. tion (11) then gives the resultant change in the parameter $m$ and the equations of reference [10] relate this change to $\Delta T$. The difficult step in this process is the initial free-energy calculation.

The other method is more direct and, though it is rather less satisfying, we shall use it here for this reason. We attempt to estimate the activation temperature of a surface composed entirely of silver atoms, and from this and the linear relation implied by equation (12) we evaluate the constant $A$.

Since we are interested in the early stages of photolysis in which the silver atoms remain approximately in the AgI lattice, the activation temperature we wish to estimate is that of a hypothetical metallic silver of wurtzite structure. At best we can only hope for a rough approximation, but uncertainties in this factor merely change the time scale without making any qualitative alterations in the deactivation behavior. Since the time scale is inversely proportional to the radiation intensity $I$, and this is itself a highly variable quantity, we shall not be concerned at uncertainties in the constant $A$ of the order of a factor 2 .

To estimate the activation temperature of our hypothetical wurtzite silver, we observe that ordinary silver is not a recognized nucleating agent and is probably only active at some temperature below $-40 \mathrm{C}$. On the other hand, InSb, which has the closely related zincblende lattice with constants close to those of AgI, but with much less polar structure, is active below about $-16 \mathrm{C}[17]$. The critical temperature we seek, thus, probably lies between about $-20 \mathrm{C}$ and $-50 \mathrm{C}$. Since bulk AgI has a critical temperature of $-4 \mathrm{C}$, this implies a total change in $\Delta T$ of $10 \mathrm{C}$ to $45 \mathrm{C}$. We shall choose $30 \mathrm{C}$ as a value which is probably not in error by more than a factor 2 .

Since the density of surface molecules of AgI is approximately $\left(1.4 \times 10^{22}\right)^{\frac{2}{3}}=6 \times 10^{14} \mathrm{~cm}^{-2}$, the constant $A$ in equation (12) has the value

$$
A=30 / 6 \times 10^{14}=5 \times 10^{-14}
$$

with units of degrees centigrade and silver atoms per square centimeter.

De-activation rate. - Now that we have found explicit expressions for all the constants involved, we can rewrite equation (13) in the form

$$
d \Delta T / d t \approx 1.4 \times 10^{-22} R^{-3} F(k, R) I,
$$

where the rate is in degrees centigrade per second, $F(k, R)$ is given by (14) and $k=10^{5}$. Inserting a specific value of $I$ for sunlight, as discussed above,

$$
d \Delta T / d t=5.1 \times 10^{-5} R^{-3} F(k, R),
$$

where the rate is now expressed in the more convenient unit of degrees centigrade per hour.

The relation given by equation (19) is plotted in fig. 2. It is seen that very small particles all decay at about 22 deg per hr, whilst the decay rate for large particles is inversely proportional to their radius. The absolute decay rates are probably correct to within about a factor of 4 .

Size distribution in smokes.-The distribution of particle sizes in smokes produced by various burners has been discussed elsewhere $[9 ; 15]$. Many smokes have an approximately normal distribution of $\log R$ 


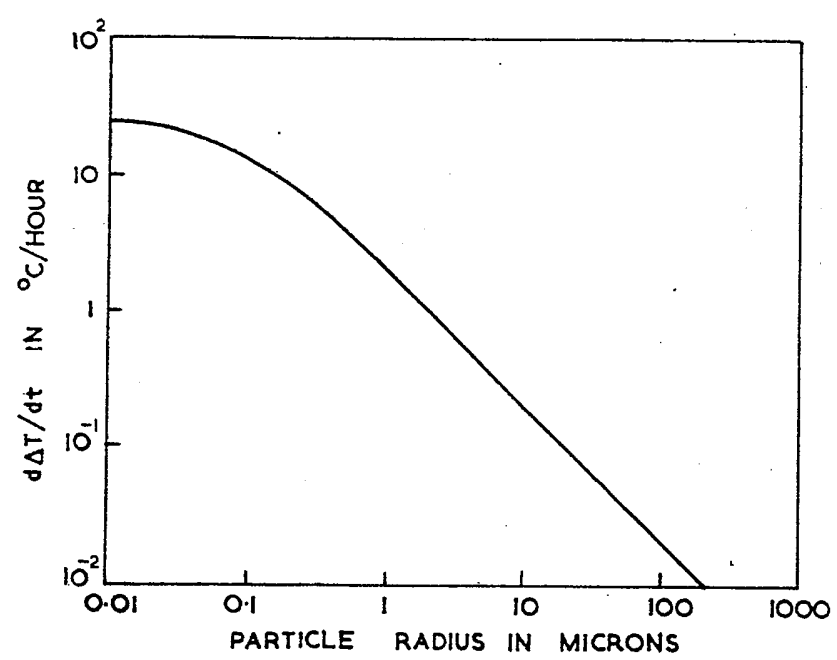

FIG. 2. Rate of increase, with time of exposure to direct sunlight, of supercooling $\Delta T$ required for nucleation of an ice crystal by a silver-iodide particle of given radius.

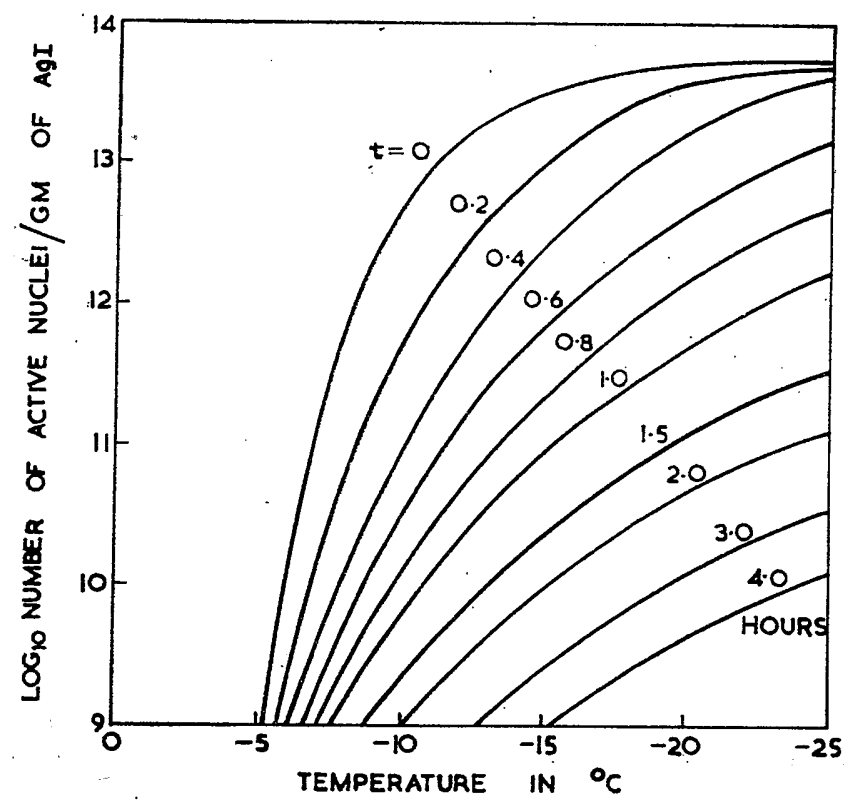

FIG. 3. Activity curves of smoke produced by kerosene burner after time $t$ of exposure to direct sunlight. Curve for $t=0$ is experimental, and other curves are derived from it theoretically.

and, to a first approximation, differ only in mean and standard deviation. Both these quantities, however, differ widely from one burner to another.

Explicit mention should here be made of an assumption usually made about these burners. This is that the smoke which they produce consists basically of silver iodide. This is open to question [16] in the case of burners which consume a solution of $\mathrm{AgI}$ and $\mathrm{NaI}$ in acetone. The experimental evidence indicates that, whatever the smoke is, it behaves just like silver iodide, having the same threshold (about $-4 \mathrm{C}$ ) and producing the number of active nuclei per gram expected of a substance active by sublimation [15]. These results lend very strong support to this assumption, which we shall agree to adopt here.

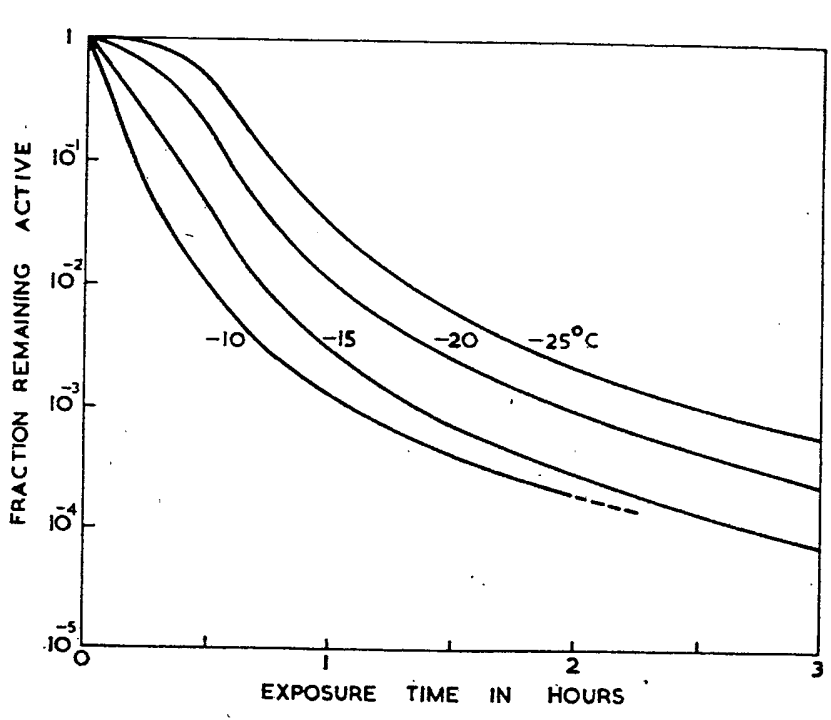

FIG. 4. Calculated decrease of nucleation activity of smoke from kerosene burner with exposure to direct sunlight. The parameter is the temperature of the supercooled cloud in which the measurement of nucleation efficiency is made.

The output of a burner is usually specified as the number of nuclei, per second, or per gram of AgI burnt, which are active at each temperature within the range of interest. From fig. 1, a size can be assigned such that all particles active at a given temperature are larger than that particular size, and with the aid of equation (13) the subsequent activity of the smoke can be calculated.

\section{Behavior of typical smokes}

Now that the decay behavior of a single particle has been found, the behavior of a heterogeneous smoke can be determined by summation over all the particles, using fig. 1 to assign sizes to particles active at a given temperature.

The activity curve is shown, in fig. 3 at $t=0$, for the smoke produced by a typical burner [18] burning AgI-NaI in acetone solution in a kerosene flame. As can be seen from the activity curve, this burner produces a wide range of rather coarse particles with relatively few of diameter less than $300 \AA$.

Curves showing the decay of the activity distribution after various times of exposure to sunlight are also shown in fig. 3 , and in fig. 4 decay curves have been plotted for several fixed temperatures. Two things should be noted about these curves. In the first place, the decay is not exponential but becomes much slower after one or two hours' exposure. In the second place, the exact form of the decay curve depends markedly upon the measurement temperature.

For this smoke, Smith and Heffernan [2] found an average decay rate, taken over two hours, of about a factor 20 per hr at $-17 \mathrm{C}$. This is in fortuitously good agreement with our calculations which would predict a decay of a factor 40 per hr when averaged over a 


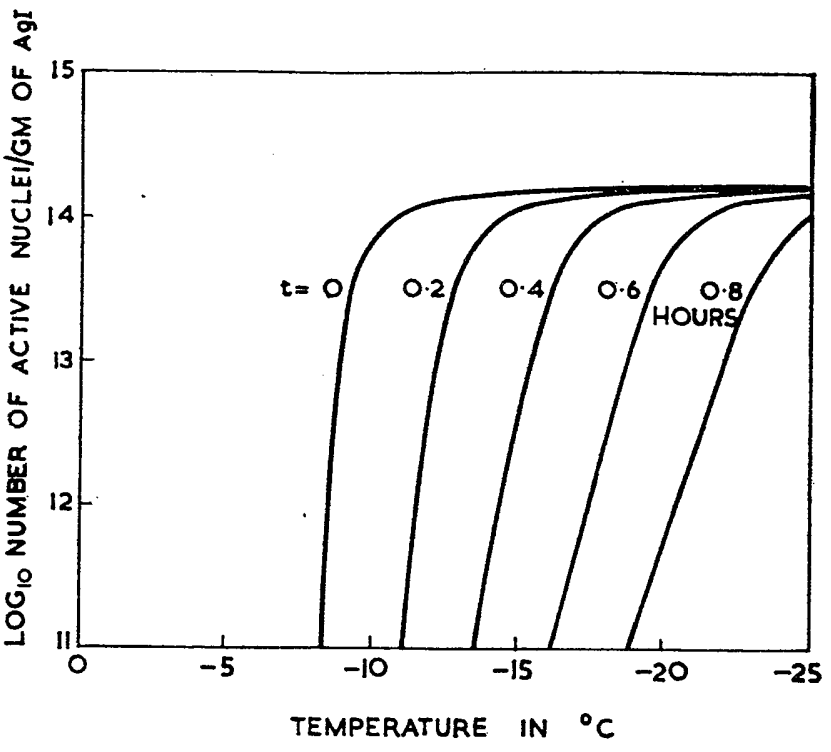

FIG. 5. Activity curves of a hypothetical smoke having narrow distribution of particle diameters around $1200 \AA$ A. Parameter is time of exposure to direct sunlight.

two-hour period at $-17 \mathrm{C}$. Nothing better than semiquantitative agreement should really be expected in view of the uncertainties in the time scale of the theory.

Smith and Heffernan also discuss a hydrogen burner which was found to have a much higher rate of decay. Their measurements indicated a decay rate of $10^{6}$ per hour at $-17 \mathrm{C}$ when measured over a half hour period. A calculation similar to that detailed above for the kerosene burner has been performed for the smoke from the hydrogen burner. This smoke is much finer than the smoke of the kerosene burner, and this leads to a somewhat greater decay rate. This, coupled with the fact that the averaging was only over the initial half-hour period leads to an "average" calculated decay rate of $10^{4}$ per hr. Whilst this is not quite as large as the observed value, the semi-quantitative agreement with the experiment is gratifying in view of the approximate nature of both the theory and the experimerts.

In fig. 5 , there is shown, at $t=0$, the computed activity curve of a smoke with a fairly uniform particle size. This gives the sharply angled curve sometimes seen in solid fuel burners. In this case, the meanparticle diameter is about $1200 \AA$. Subsequent activity curves are shown in fig. 5 , and in fig. 6 the decay curves at various temperatures are plotted.

The decay curves in this case differ greatly from those of a coarsely heterogeneous smoke, and illustrate the behavior reported by Inn [5] and by Birstein [8], who noted an initial small rate of decay followed rapidly by almost complete destruction of the nucleating power of the smoke. The time scale depends critically upon the temperature at which the experiment is made, but again is in good agreement with

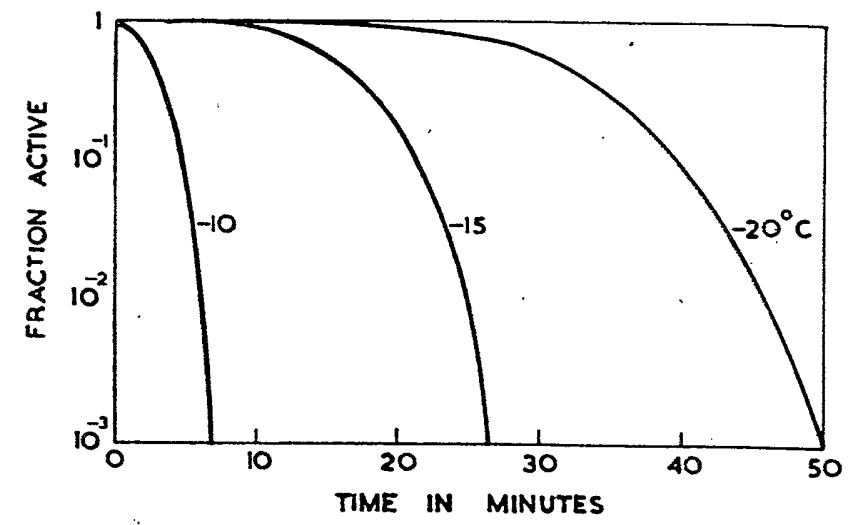

FIG. 6. Decay curves for the hypothetical smoke of fig. 5 . Parameter is temperature of the supercooled cloud in which the measurement is made.

the reported results of 5 to $150 \mathrm{~min}$ for complete de-activation.

The work of Vonnegut and Neubauer [6] alone disagrees with the predictions of our theory. They reported negligible decay after $8 \mathrm{hr}$ of irradiation with an ultraviolet lamp of intensity comparable with that of sunshine. Because of the large time involved, it is difficult to explain this as a size effect, and it seems that some form of desensitization may have been present, as will be discussed below.

\section{Environmental factors}

In the simple theory we have discussed at length, the approximation was made of neglecting any traps of a specifically surface nature. This allowed us to put $\gamma_{0}=0$ in (15) and greatly simplified the subsequent calculations.

In practice, such surface traps arise principally by reaction with the environment. For example, active gases in the atmosphere may attack the surface and create new trapping centers. This may be of principal importance within the flame of the burner itself where the surface reactivity is enhanced by the high temperature.

Another form of interaction with the environment may be localized at imperfect areas of the surface. This will be the case for the less reactive constituents of the environment, and the centers of attack may be just the centers responsible for electron trapping and the production of surface silver. Depending upon the chemical nature of the attacking substance, the activity of the traps may be either increased or decreased, so that the subsequent decay rate may be raised or lowered.

Experimental data upon reaction with the environment is limited, though some work has been done by Reynolds et al, [7] on the effects of ammonia, by Birstein [8] on the effects of humidity, and by Sano and Fukuta [19] on the effects of these substances and 
of hydrogen sulfide. Too little is yet known of the details of the trapping process for useful comment to be made on these results.

\section{Conclusion}

The photo de-activation of silver iodide as an icecrystal nucleus has been ascribed to the production of photolytic silver at trapping centers in the volume and on the surface of the nucleating particle. Silver produced at the surface raises the free energy of the interface between the nucleus and ice and so reduces the activity of the particle. This sort of behavior is to be expected for all substances which can be decomposed photolytically.

Applying the theory explicitly to silver iodide, the decay behavior was found to depend critically upon the particle-size distribution within the smoke, and upon the temperature at which measurements were carried out. Specific examples calculated gave good agreement with the experiments, and in other cases the general behavior was in agreement with that found experimentally.

\section{REFERENCES}

1. Smith, E. J., K. J. Heffernan, and B. K. Seely, 1955: The decay of ice-nucleating properties of silver iodide in the atmosphere. J. Meteor., 12, 379-385.

2. Smith, E. J., and K. J. Heffernan, 1956: The decay of the ice nucleating properties of silver iodide released from a mountain top. Quart. J. r. meteor. Soc., 82, 301-309.

3. Sriith, E. J., K. J. Heffernan, and W. J. Thomson, 1958: The decay of the ice nucleating properties of silver iodide released from an aircraft. Quart. J. r. meteor. Soc., 84, 162-165.

4. Reynolds, S. E., W. Hume, B. Vonnegut, and V. J. Schaefer, 1951: Effect of sunlight on the action of silver iodide particles as sublimation nuclei. Bull. Amer. meteor. Soc., $32,47$.

5. Inn, E. C. Y., 1951: Photolytic inactivation of ice forming silver iodide nuclei. Bull. Amer. meteor. Soc., 32, 132-135.

6. Vonnegut, B., and R. Neubauer, 1951: Recent experiments on the effect of ultraviolet light on silver iodide nuclei. Bull. Amer. meteor. Soc., 32, 356.

7. Reynolds, S. E., W. Hume, and M. McWhirter, 1952: Effects of sunlight and ammonia on the action of silver iodide particles as sublimation nuclei. Bull. Amer. meteor. Soc., 33, 26-31.

8. Birstein, S. J., 1952: The effect of relative humidity on the nucleating properties of photolysed silver iodide. Bull. Amer. meteor. Soc., 33, 431-434.

9. Fletcher, N. H., 1959: On ice-crystal production by aerosol particles. J. Meteor., 16, 175-182.

10. Fletcher, N. H., 1958: Size effect in heterogeneous nucleation. J. Chem. Phys., 29, 572-576.

11. Mitchell, J. W., 1955: The photographic process. Chemistry of the solid state, ed. by W. E. Garner, London, Butterworth's Publications, 311-335.

12. Van de Hulst, H. C., 1957 : Light scattering by small particles. New York, Wiley Pub. Co., 276.

13. Seitz, F., 1951: Speculations on the properties of the silver halide crystals. Revs. Mod. Phys., 23, 328-352.

14. Koller, L. R., 1952: Ultravialet radiation, New York, Wiley Pub. Co., 106-139.

15. Fletcher, N. H., 1959: Optimum performance of silver iodide smoke generators. J. Meteor., to be publ. Aug. 1959.

16. Mason, B. J., and J. Hallett, 1956: Artificial ice-forming nuclei. Nature, 177, 681-683.

17. Pruppacher, H. R., and R. Sänger, 1955 : Mechanismus der Vereisung unterkühlter Wassertropfen durch disperse Keimsubstanzen. Zeits. f. ang. Math u. Phys., 6, 407-416.

18. Smith, E. J. and K. J. Heffernan, 1954 : Airborne measurements of the concentration of natural and artificial freezing nuclei. Quart. J. r. meteor. Soc., 80, 182-197.

19. Sano, I., and N. Fukuta, 1956: Effects of water vapour, ammonia and hydrogen sulphide against the decay of silver iodide smoke under irradiation of ultraviolet light. J. meteor. Soc. Japan, Series II, 34, 34-40. 